Chronic Obstructive Pulmonary Diseases: Journal of the COPD Foundation

\author{
Original Research
}

\title{
Pulmonary Subtypes Exhibit Differential Global Initiative for Chronic Obstructive Lung Disease Spirometry Stage Progression: The COPDGene ${ }^{\circledR}$ Study
}

Kendra A. Young, $\mathrm{PhD}^{1}$ Mathew J. Strand, $\mathrm{PhD}^{2}$ Margaret F. Ragland MD, MS ${ }^{3}$ Gregory L. Kinney, $\mathrm{PhD}^{1}$ Erin E. Austin, $\mathrm{PhD}^{4}$ Elizabeth A. Regan, $\mathrm{MD}, \mathrm{PhD}^{5}$ Katherine E. Lowe, $\mathrm{MSc}^{1}$ Barry J. Make, $\mathrm{MD}^{5}$ Edwin K. Silverman, MD, $\mathrm{PhD}^{6}$ James D. Crapo, $\mathrm{MD}^{5}$ John E. Hokanson, $\mathrm{PhD}^{1}$ for the COPDGene Investigators

\begin{abstract}
Rationale: We classified individuals into pulmonary disease subtypes based on 2 underlying pathophysiologic disease axes (airway-predominant and emphysema-predominant) and their increased mortality risk. Our next objective was to determine whether some subcomponents of these subtypes are additionally associated with unique patterns of Global initiative for chronic Obstructive Lung Disease (GOLD) spirometry stage progression. Methods: After accounting for intra-individual measurement variability in spirometry measures between baseline (Phase 1) and the 5-year follow up (Phase 2) of the COPD Genetic Epidemiology (COPDGene ${ }^{\circledR}$ ) study, 4615 individuals had complete data that would characterize patterns of disease progression over 5 years (2033 non-Hispanic whites; 827 African Americans; 48\% female). Individuals could express increased risk for mortality on one or both of the primary subtype axes (airway-predominant or emphysema-predominant) and thus they were further classified into 6 groups: high-risk airway-predominant disease only (APD-only), moderate-risk airway-predominant disease only (MR-APD-only), high-risk emphysema-predominant disease only (EPD-only), combined high-risk airway- and emphysema-predominant disease (combined APD-EPD), combined moderate-risk airway- and emphysema-predominant disease (combined MR-APD-EPD), and no highrisk pulmonary subtype. Outcomes were dichotomized for GOLD spirometry stage progression from Phase 1 to Phase 2. Logistic regression of the progression outcomes on the pulmonary subtypes were adjusted for age, sex, race, and change in smoking status.

Results: The MR-APD-only group was associated with conversion from GOLD 0 to preserved ratio-impaired spirometry (PRISm) status (odds ratio [OR] 11.3, 95\% confidence interval [CI] 5.7-22.1) and GOLD 0 to GOLD 2-4 (OR 6.0, 95\% CI 2.0-18.0). The EPD-only group was associated with conversion from GOLD 0 to GOLD 1 (OR 2.4, 95\% CI 1.2-4.6), and GOLD 1 to GOLD 2-4 (OR 2.6, 95\% CI 1.0-6.9). Conversion between PRISm and GOLD 2-4 (31\%-38\%) occurred in both the APD-only and the MR-APD-only groups.

Conclusion: Differential conversion occurs from GOLD 0 to PRISm and GOLD 0 to GOLD 1 based on groups expressing airway-predominant disease or emphysema-predominant disease independently or in combination. Airway-predominant and emphysema-predominant subtypes are highly important in determining patterns of early disease progression.
\end{abstract}

Abbreviations: Global initiative for chronic Obstructive Lung Disease, GOLD; COPD Genetic Epidemiology, COPDGene ${ }^{\circledR}$ high-risk airway-predominant disease only, APD-only; moderate-risk airway-predominant disease only, MR-APD-only; high-risk emphysemapredominant disease only, EPD-only; combined high-risk airway and emphysema-predominant disease, combined APD-EPD; combined moderate-risk airway and emphysema-predominant disease, combined MR-APD-EPD; preserved ratio-impaired spirometry, PRISm; odds 
ratio, OR; confidence interval, CI; chronic obstructive pulmonary disease, COPD; computed tomography, CT; 6-minute walk distance, 6MWD; longitudinal follow-up program, LFU; wall area percentage, WA\%; Hounsfield units, HU; forced expiratory volume in 1 second, FEV 1; forced vital capacity, FVC; preserved ratio-impaired spirometry, PRISm; body mass index, BMI; standard deviation, SD; percentage low attentuation of area, LAA\%; analysis of variance, ANOVA

Funding Support: The COPDGene ${ }^{\circledR}$ study is funded by the National Heart, Lung, and Blood Institute grants U01 HL089897 and U01 HL089856. The COPDGene ${ }^{\circledR}$ study is also supported by the COPD Foundation through contributions made to an Industry Advisory Committee comprised of AstraZeneca, Boehringer-Ingelheim, Genentech, GlaxoSmithKline, Novartis, Pfizer, Siemens, and Sunovion.

Date of Acceptance: October 29, 2019

Citation: Young KA, Strand MJ, Ragland MF, et al for the COPDGene ${ }^{\circledast}$ Investigators. Pulmonary subtypes exhibit differential Global Initiative for Chronic Obstructive Lung Disease spirometry stage progression: the COPDGene study. Chronic Obstr Pulm Dis. 2019;6(5):414-429. doi: https://doi.org/10.15326/jcopdf.6.5.2019.0155

1 Department of Epidemiology, Colorado School of Public Health, University of Colorado Anschutz Medical Campus, Aurora

2 Division of Biostatistics and Bioinformatics, Office of Academic Affairs, National Jewish Health, Denver, Colorado

3 Department of Pulmonary Sciences and Critical Care Medicine, University of Colorado School of Medicine, Aurora

4 Department of Mathematical and Statistical Sciences, University of Colorado at Denver

5 Department of Medicine, National Jewish Health, Denver, Colorado

6 Channing Division of Network Medicine, Brigham and Women's Hospital, Boston, Massachusetts

\section{Address correspondence to:}

Kendra A. Young, MSPH, PhD

Department of Epidemiology

Colorado School of Public Health

University of Colorado Anschutz Medical Campus

13001 East 17th Avenue

Room W3142, Campus Box B-119

Aurora, CO 80045

Phone: 303-724-4441

Email: Kendra.Young@cuanschutz.edu

\section{Keywords:}

chronic obstructive pulmonary disease; COPD; progression; subtypes; spirometry; airway-predominant; emphysemapredominant; COPD Genetic Epidemiology study; COPDGene ${ }^{\circledR}$

\section{Introduction}

Chronic obstructive pulmonary disease (COPD) is a heterogeneous disorder. ${ }^{1}$ The Global initiative for chronic Obstructive Lung Disease (GOLD) guidelines ${ }^{2}$ are currently used to grade the severity of spirometric disease. Previous studies have indicated that lung function declines and progression occurs across GOLD stages, ${ }^{3-6}$ although decline varies and many individuals remain stable. While smokers without spirometrically defined COPD commonly show indications of lung impairment and disease, ${ }^{7,8}$ the risk and pattern of progression to spirometrically defined COPD under GOLD stages is unclear. Previous studies have indicated that only $20 \%$ progress $^{9}$ to defined COPD, but that may be an underestimation of the true number. ${ }^{10,11}$ Progression from preserved spirometry may depend on subtypes ${ }^{12}$ or clinical phenotypes such as increased air trapping. ${ }^{13,14}$

Recently, we identified continuous pulmonary disease axes based on the correlational structure of Phase 1 chest computed tomography (CT) and pulmonary function tests that were associated with mortality risk. ${ }^{15}$ The pattern of risk indicated a complex relationship, and we further classified individuals into high-risk pulmonary subtype groups based on mortality risk within the 2 underlying pathophysiologic disease axes (airway-predominant disease [APD] and emphysema-predominant disease [EPD]). ${ }^{16}$ These groups were associated with mortality independent of GOLD spirometry stage, and they appear to represent different disease pathways. We found that for the emphysema-predominant axis, marked increased risk for mortality occurred in the highest 2 deciles of the axis. In contrast, the airway-predominant axis showed a more robust differentiation of mortality risk with the top $60 \%$ showing an enhanced hazard ratio and the top 2 deciles the greatest increase in mortality hazard ratio (Figure 1).

Because some high-risk individuals express characteristics of both the airway-predominant and emphysema-predominant subtypes, a further subdivision was done to create 6 groups that share common characteristics in terms of mortality risk and their relationship to the airway-predominant and emphysema-predominant subtypes. Our objective in the current analysis is to determine whether these high-risk groups are uniquely associated with GOLD spirometry stage progression. 


\section{Figure 1. Cox Proportional Hazards Regression Was Used to Assess Mortality Risk Across Deciles of Both the Emphysema-Predominant and Airway-Predominant Disease Axes}
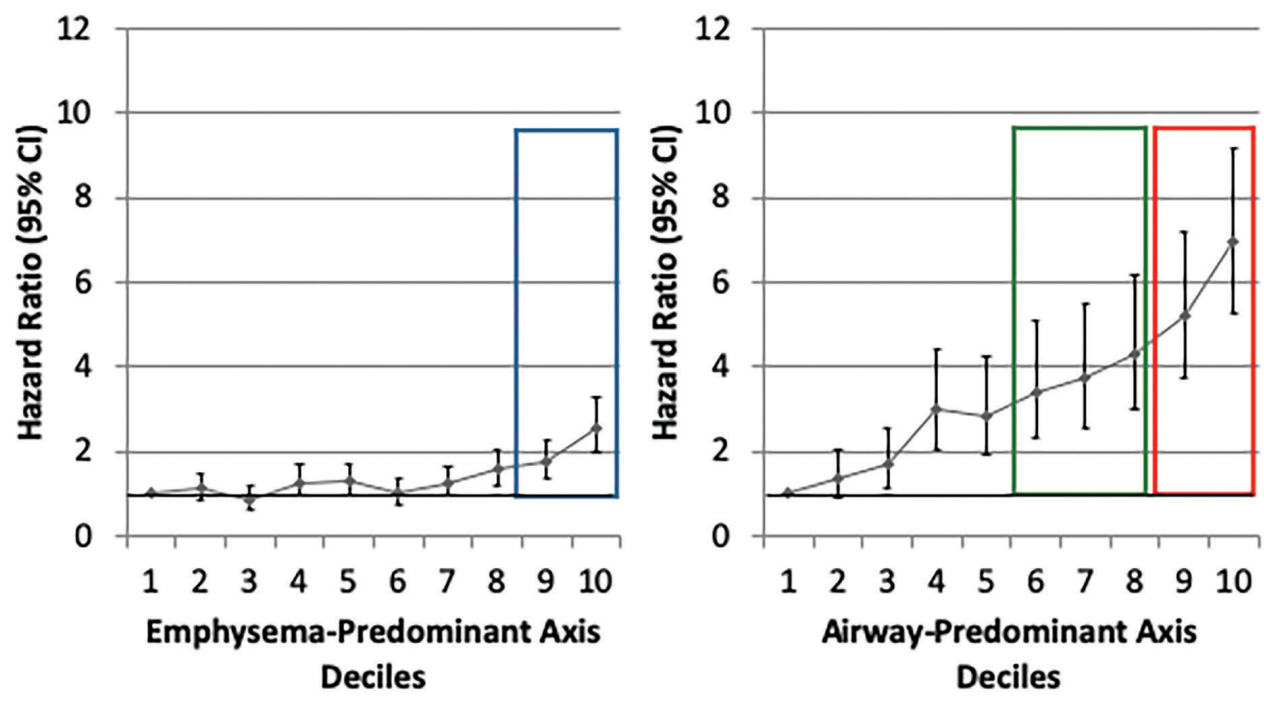

The model included age, sex, race, pack years of smoking, current smoking status, body mass index, high-blood pressure, a square term for the airway disease axis factor score (to account for part of the non-linear association with mortality), the gas trapping axis, the computed tomography intensity variability axis, and the total lung capacity and functional residual capacity axis. The 2 upmost deciles (red and blue boundaries) of both the emphysema-predominant and airway-predominant axis were used to define the high-risk groups. The sixth, seventh and eighth deciles (green boundaries) were used to define the moderate-risk airway-predominant group.

\section{Methods}

\section{COPD Genetic Epidemiology Study}

The Genetic Epidemiology of COPD (COPDGene ${ }^{\circledR}$ ) study (www.copdgene.org) is a longitudinal multicenter observational study designed to identify genetic and epidemiologic factors associated with the development of COPD and to characterize disease progression using pulmonary function tests and volumetric CT scans. ${ }^{7}$ The COPDGene ${ }^{\circledast}$ study recruited 10,198 non-Hispanic whites and African Americans aged 45-80 years at 21 clinical centers across the United States beginning in 2008. Of the 10,198 participants enrolled at Phase 1 (baseline), 8157 had complete data required to assess disease axes. ${ }^{15}$ Of the 8157 Phase 1 participants, 4615 (3301 non-Hispanic whites and 1314 African Americans; 49\% female) completed a 5-year Phase 2 follow-up with complete pulmonary subtype data and a defined GOLD spirometry stage at both Phase 1 and Phase 2 (Table 1). In order to assess disease-related progression to a different GOLD stage, we removed individuals who were sufficiently close to a GOLD boundary in Phase 1 that change across the GOLD boundary could likely be attributed to intra-individual measurement variability alone. Thus, 1755 individuals were removed from the Phase 2 progression analysis to reduce the impact of this random variability (see detailed methods below), leaving 2860 individuals for analysis. Descriptive characteristics of the study population stratified by these high-risk groups are reported in Table 2 .

The COPDGene ${ }^{\circledR}$ cohort is made up of individuals with a heavy smoking history. All were current and former smokers with least 10 pack years of smoking history; however, the mean smoking history for all groups ranged from 37-53 pack years. ${ }^{17}$ The COPDGene ${ }^{\circledast}$ study was approved by the institutional review board at each center, and all participants provided written informed consent. In addition, all protocols were approved by the institutional review boards at National Jewish Health and the University of Colorado-Denver. Individuals underwent detailed baseline characterization, including demographics; anthropometrics; medical and smoking history; preand post-bronchodilator spirometry as well as 6-minute 


\section{Table 1. Phase 2 Status by Subtype}

\begin{tabular}{|c|c|c|c|c|c|c|}
\hline \multirow[b]{2}{*}{ Group } & \multirow{2}{*}{$\begin{array}{c}\text { Phase } 1 \\
n\end{array}$} & \multicolumn{5}{|c|}{ Phase 2} \\
\hline & & $\begin{array}{l}\text { Individuals } \\
\text { with Full } \\
\text { Follow-up } \\
\text { Data }\end{array}$ & Mortality ${ }^{\mathrm{a}}$ & $\begin{array}{l}\text { Missing at } \\
\text { Follow-up }\end{array}$ & $\begin{array}{l}\text { Individuals } \\
\text { Removed } \\
\text { Due to } \\
\text { Measurement } \\
\text { Variability }\end{array}$ & $\begin{array}{c}\text { Individuals } \\
\text { in Phase } 2 \\
\text { Progression } \\
\text { Analyses }\end{array}$ \\
\hline APD-Only & 1007 & 477 (47.4\%) & $218(21.6 \%)$ & $312(31.0 \%)$ & 98 (9.7\%) & $379(37.6 \%)$ \\
\hline MR APD-Only & 1995 & $1100(55.1 \%)$ & $219(11.0 \%)$ & $676(33.9 \%)$ & $679(34.0 \%)$ & $421(21.1 \%)$ \\
\hline EPD-Only & 554 & $351(63.4 \%)$ & $50(9.0 \%)$ & $153(27.6 \%)$ & $128(23.1 \%)$ & $223(40.3 \%)$ \\
\hline Combined APD-EPD & 625 & $197(31.5 \%)$ & $297(47.5 \%)$ & $131(21.0 \%)$ & $3(0.5 \%)$ & $194(31.0 \%)$ \\
\hline Combined MR APD-EPD & 452 & $252(55.7 \%)$ & $104(23.0 \%)$ & $96(21.2 \%)$ & $13(2.9 \%)$ & $239(52.9 \%)$ \\
\hline No HR or MR Group & 3524 & $2238(63.5 \%)$ & $215(6.1 \%)$ & $1071(30.4 \%)$ & $834(23.7 \%)$ & $1404(39.8 \%)$ \\
\hline Totals & 8157 & 4615 & 1103 & 2439 & 1755 & 2860 \\
\hline
\end{tabular}

aMortality is the number of participants who died prior to a Phase 2 visit (approximately a five-year mortality).

Percentages represent the distribution by group.

APD-only=high risk airway-predominant disease only; MR APD-only=moderate risk airway-predominant disease only;

EPD-only=high risk emphysema; No HR or MR group=no high risk or moderate risk group

walk distance (6 MWD) testing according to American Thoracic Society standards ${ }^{18,19}$; and inspiratory and expiratory CT scans using a standardized protocol. ${ }^{7}$ Enrollment in Phase 1 of the COPDGene ${ }^{\circledR}$ study took place between 2008 and 2011, and participants were prospectively followed every 6 months by telephone and web-based inquiry as part of a longitudinal followup program (LFU) to determine mortality, comorbid disease events and disease status. ${ }^{20}$ Participants enrolled in Phase 1 were invited to participate in a 5-year follow-up study visit (Phase 2, 2012-2016).

Thoracic CT scans were acquired at full inspiration at both Phase 1 and Phase 2 using the same standardized protocol. ${ }^{7}$ Quantification of airway wall thickness, emphysema and gas trapping was performed by Thirona (Thirona, The Netherlands). Lung and airways structures were automatically extracted from inspiratory CT scans and visually approved by trained analysts. Airway wall thickness was assessed using wall area percentage (WA\%) for segmental airways. $^{21,22}$ In this analysis we report WA\% which we found to be the most robust airway measurement for our primary assessment of airway disease. Emphysema was defined as the percentage of low-attenuation area below -950 Hounsfield units (HU) (\% emphysema). ${ }^{23}$

Current smoking status and pack years of smoking were determined by questionnaire. COPD was grouped as spirometric grades $1-4$ based on the GOLD guidelines. ${ }^{2}$ Participants without spirometric evidence of airflow obstruction (forced expiratory volume in 1 second $\left[\mathrm{FEV}_{1}\right]$ to force vital capacity $[F V C]$ ratio $\geq 0.70$ and $\mathrm{FEV}_{1} \geq 80 \%$ predicted) were classified as GOLD 0. Participants with FEV $1 / F V C \geq 0.70$ and $\mathrm{FEV}_{1}<80 \%$ predicted were classified as preserved ratio-impaired spirometry (PRISm). ${ }^{8}$

\section{Mortality Ascertainment}

Vital status (mortality assessed through January 31,2018 ) was determined through the COPDGene ${ }^{\circledR}$ participant tracking LFU program and augmented with searching the Social Security Death Index on the subset of participants with available social security numbers. Results were aggregated centrally. Participant follow-up time was the time between their Phase 1 study visit and identified death or most recent active LFU participation. Mortality for these analyses was limited to those who died between Phase 1 and Phase 2 ( 5 years) to account for their removal from the progression analyses.

\section{Identification of High-Risk Groups Based on the Airway-Predominant and Emphysema- Predominant Disease Axes}

Factor analysis of 26 spirometry and CT variables were used to classify 8157 COPDGene ${ }^{\circledR}$ participants onto disease axes. The loading of the variables on the 


\section{Table 2. Phase 1 Characteristics of Participants in Phase Two Progression Analysis - By Group}

\begin{tabular}{|c|c|c|c|c|c|c|c|}
\hline Characteristic & $\begin{array}{c}\text { APD-Only } \\
n=379\end{array}$ & $\begin{array}{c}\text { MR } \\
\text { APD-Only } \\
n=421\end{array}$ & $\begin{array}{c}\text { EPD-Only } \\
n=223\end{array}$ & $\begin{array}{c}\text { Combined } \\
\text { APD-EPD } \\
n=194\end{array}$ & $\begin{array}{l}\text { Combined } \\
\text { MR } \\
\text { APD-EPD } \\
n=239\end{array}$ & $\begin{array}{c}\text { No HR or } \\
\text { MR Group } \\
n=1404\end{array}$ & $P$ value \\
\hline Age (mean $\pm \mathrm{SD})$ & $60.3 \pm 8.4$ & $59.9 \pm 8.7$ & $60.4 \pm 8.5$ & $63.7 \pm 7.7$ & $65.0 \pm 7.3$ & $58.6 \pm 8.6$ & $<0.0001$ \\
\hline Sex (Female) $(\mathrm{n}, \%)$ & $175(46.2 \%)$ & $194(46.1 \%)$ & $98(44.0 \%)$ & $82(42.3 \%)$ & $131(54.8 \%)$ & $684(48.7 \%)$ & $<0.0001$ \\
\hline Race $(\mathrm{AA})(\mathrm{n}, \%)$ & $98(25.9 \%)$ & $120(28.5 \%)$ & 77 (34.5\%) & $53(27.3 \%)$ & $55(23.0 \%)$ & $424(14.8 \%)$ & $<0.0001$ \\
\hline \multicolumn{8}{|l|}{ BMI Category $(\mathrm{n}, \%)$} \\
\hline$<20$ & $9(2.4 \%)$ & $17(4.0 \%)$ & $8(3.6 \%)$ & $16(8.3 \%)$ & $17(4.0 \%)$ & $35(2.5 \%)$ & \\
\hline $20-25$ & $56(14.8 \%)$ & $86(20.4 \%)$ & $84(37.7 \%)$ & $59(30.4 \%)$ & $82(34.3 \%)$ & $358(25.5 \%)$ & $<0.0001$ \\
\hline$>25$ & $314(82.9 \%)$ & 318 (75.5\%) & $131(58.7 \%)$ & $119(61.3 \%)$ & $140(58.6 \%)$ & $1011(72.0 \%)$ & \\
\hline \multicolumn{8}{|l|}{ GOLD Stage: $(\mathrm{n}, \%)$} \\
\hline PRISm & $75(19.8 \%)$ & $92(21.7 \%)$ & $3(1.4 \%)$ & $0(0.0 \%)$ & $0(0.0 \%)$ & $9(0.6 \%)$ & \\
\hline 0 & $0(0.0 \%)$ & 57 (13.5\%) & $102(45.7 \%)$ & $0(0.0 \%)$ & $0(0.0 \%)$ & $1216(86.6 \%)$ & \\
\hline 1 & $0(0.0 \%)$ & $8(1.9 \%)$ & $36(16.1 \%)$ & $0(0.0 \%)$ & $0(0.0 \%)$ & $130(9.3 \%)$ & $<0.0001$ \\
\hline 2 & $96(25.3 \%)$ & 239 (56.5\%) & $82(36.8 \%)$ & $3(1.2 \%)$ & $102(43.0 \%)$ & $49(3.5 \%)$ & \\
\hline 3 & $188(49.6 \%)$ & $27(6.4 \%)$ & $0(0.0 \%)$ & 105 (54.1\%) & 135 (57.0\%) & $0(0.0 \%)$ & \\
\hline 4 & $20(5.3 \%)$ & $0(0.0 \%)$ & $0(0.0 \%)$ & $86(44.3 \%)$ & $0(0.0 \%)$ & $0(0.0 \%)$ & \\
\hline $\begin{array}{l}\text { FEV } \mathbf{1} \% \text { Predicted } \\
(\text { mean } \pm \mathrm{SD})\end{array}$ & $48.3 \pm 11.5$ & $68.1 \pm 12.6$ & $90.8 \pm 21.4$ & $31.3 \pm 8.3$ & $48.8 \pm 8.2$ & $102.4 \pm 11.0$ & $<0.0001$ \\
\hline $\begin{array}{l}\mathbf{F E V}_{\mathbf{1}} / \mathbf{F V C} \\
(\text { mean } \pm \mathrm{SD})\end{array}$ & $0.57 \pm 0.14$ & $0.64 \pm 0.12$ & $0.66 \pm 0.13$ & $0.37 \pm 0.08$ & $0.46 \pm 0.09$ & $0.77 \pm 0.07$ & $<0.0001$ \\
\hline $\begin{array}{l}\text { Emphysema } \\
\text { (LAA\% } \leq-950 \mathrm{HU})\end{array}$ & $4.0 \pm 4.9$ & $3.8 \pm 4.3$ & $14.1 \pm 8.8$ & $25.6 \pm 11.7$ & $22.6 \pm 10.1$ & $2.3 \pm 2.6$ & $<0.0001$ \\
\hline $\begin{array}{l}\text { Gas Trapping } \\
(\mathrm{LAA} \% \leq-856 \mathrm{HU})\end{array}$ & $24.2 \pm 16.4$ & $21.6 \pm 15.1$ & $29.2 \pm 16.1$ & $58.2 \pm 12.2$ & $51.8 \pm 13.4$ & $11.8 \pm 9.6$ & $<0.0001$ \\
\hline $\begin{array}{l}\text { Wall Area } \\
\text { Percentage } \\
\text { (segmental airways) }\end{array}$ & $60.0 \pm 6.6$ & $54.8 \pm 7.1$ & $44.0 \pm 6.5$ & $55.1 \pm 7.0$ & $51.6 \pm 6.4$ & $45.7 \pm 6.8$ & $<0.0001$ \\
\hline $\begin{array}{l}\text { Current Smoker } \\
\text { (Yes) }(\mathrm{n}, \%)\end{array}$ & $215(56.7 \%)$ & $243(57.7 \%)$ & $85(38.1 \%)$ & $45(23.2 \%)$ & $57(23.9 \%)$ & $711(50.6 \%)$ & $<0.0001$ \\
\hline $\begin{array}{l}\text { Pack Years } \\
(\text { mean } \pm \mathrm{SD})\end{array}$ & $52.1 \pm 25.8$ & $48.6 \pm 24.5$ & $41.6 \pm 22.8$ & $53.4 \pm 25.0$ & $51.5 \pm 24.7$ & $37.4 \pm 21.0$ & $<0.0001$ \\
\hline $\begin{array}{l}\text { Distance Walked } \\
\text { (feet) (mean } \pm \mathrm{SD} \text { ) }\end{array}$ & $1184.3 \pm 352.5$ & $1349.2 \pm 332.1$ & $1536.3 \pm 390.6$ & $1103.0 \pm 314.6$ & $1231.3 \pm 348.3$ & $1569.5 \pm 344.5$ & $<0.0001$ \\
\hline $\begin{array}{l}\text { SGRQ Total } \\
(\text { mean } \pm \mathrm{SD})\end{array}$ & $42.3 \pm 20.6$ & $30.8 \pm 21.9$ & $19.2 \pm 19.6$ & $47.4 \pm 18.4$ & $36.6 \pm 17.8$ & $14.3 \pm 16.2$ & $<0.0001$ \\
\hline
\end{tabular}

25 individuals are missing 6-minute walk distance

APD-only=high-risk airway-predominant disease only; MR APD-only=moderate-risk airway-predominant disease only; EPD-only=high risk emphysema; $\mathrm{HR}=$ high risk; $\mathrm{MR}=$ moderate risk; $\mathrm{BMI}=$ body mass index; $\mathrm{GOLD}=\mathrm{Global}$ initiative for chronic Obstructive Lung Disease; $\mathrm{PRISm}=$ preserved ratio-impaired spirometry; $\mathrm{FEV}_{1}$ =forced expiratory volume in 1 second; $\mathrm{FVC}=$ forced vital capacity; $\mathrm{SD}=$ standard deviation; LAA\%=percentage low attenuation area; HU=Hounsfield units;SGRQ=St George's Respiratory Questionnaire; 
axes described different disease processes. Two of these disease processes, the emphysema-predominant and airway-predominant axes, had increased mortality (Figure 1); in addition, a synergistic interaction between these 2 disease axes indicated that mortality was significantly increased at higher values of both axes in combination. ${ }^{15}$

To account for the non-linear association with mortality observed in our previous work and to determine if the association remained significant after adjustment for additional covariates, a Cox proportional hazards regression was used to assess mortality risk across deciles of both the emphysemapredominant and airway-predominant axes. The model included age, sex, race, pack years of smoking, current smoking status, body mass index (BMI), highblood pressure, a square term for the airway disease axis factor score (to account for part of the non-linear association with mortality), the gas trapping axis, the CT intensity variability axis, and the total lung capacity and functional residual capacity axis. ${ }^{15}$ While increased risk of mortality was observed across most deciles of the airway-predominant axis, the 9th and 10th deciles had the highest risk of mortality. The next 3 deciles (6th, 7th and 8th) also showed an increased mortality hazard ratio (Figure 1). Across the emphysema-predominant axis, increased mortality was observed only in the 9th and 10th deciles of the emphysema-predominant factor. Based on these results, individuals were classified into 6 high-risk groups (Figure 2; Supplementary Figures S1-S6):

- APD-Only: High-risk airway-predominant disease -participants in the 9th or 10th deciles of the airway-predominant axis who are not also in the 9th or 10th deciles of the emphysema-predominant axis.

- MR APD-only: Moderate-risk airway-predominant disease only-participants in the 6th, 7 th or 8 th deciles of the airway-predominant axis and who are not also in the 9th or 10th deciles of the emphysemapredominant axis.

- EPD-only: High-risk emphysema-predominant disease only-participants in the 9th or 10th deciles of the emphysema-predominant axis who are not also in the 9th or 10th deciles of the airwaypredominant axis.

- Combined APD-EPD: Combined high-risk airway and emphysema-predominant disease- participants in the 9th or 10th deciles of the airwaypredominant axis who are also in 9th or 10th deciles of the emphysema-predominant axis.

- Combined MR APD-EPD: Combined moderaterisk airway and emphysema-predominant diseaseparticipants in the 6th, 7 th or 8 th deciles of the airway-predominant axis and who are also in the 9th or 10th deciles of the emphysema-predominant axis.

- No HR or MR: No high-risk or moderate-risk pulmonary group-participants not in the 6th to 10th deciles of the airway-predominant axis and also not in the 9th or 10th deciles of the emphysemapredominant axis.

\section{Statistical Analyses}

Statistical analyses were performed using SAS 9.4 (SAS Institute, Cary, North Carolina). P-values less than 0.05 were considered statistically significant. Data are presented as means (standard deviation [SD]), $\mathrm{n}$ (percentages [\%]), or odds ratios (OR) and 95\% confidence intervals (CI) where appropriate. Phase 1 demographic and clinical characteristics between groups were compared with the chi-square test for categorical variables and one-way analysis of variance (ANOVA) for continuous variables.

Change in smoking status between Phase 1 and Phase 2 was classified into 4 categories: currentcurrent, former-former, current-former, and formercurrent. Outcomes were dichotomized as yes/no for GOLD spirometry stage progression from Phase 1 to Phase 2 for:

1) GOLD 0 to PRISm (FEV $1 \%$ predicted $<80 \%$, $\mathrm{FEV}_{1} / \mathrm{FVC}>0.70$ );

2) GOLD 0 to GOLD 1;

3) GOLD 0 to GOLD 2-4;

4) PRISm to GOLD 2-4;

5) GOLD 1 to GOLD 2-4.

Due to the small number of individuals in the comparison group (No HR or MR) in PRISm ( $n=9$ ), multivariable regression models were not run for PRISm to GOLD 2-4 for this group. Therefore, we used 4 logistic regression models of the progression outcomes on the high-risk and moderate-risk groups to calculate OR and $95 \%$ Cis compared to the No HR MR group after adjustment for age, sex, race, and change in smoking status. Due to a priori hypotheses, we did not correct for multiple testing. 


\title{
Figure 2. Distribution of High- and Moderate-Risk Subtypes by Pulmonary Function
}

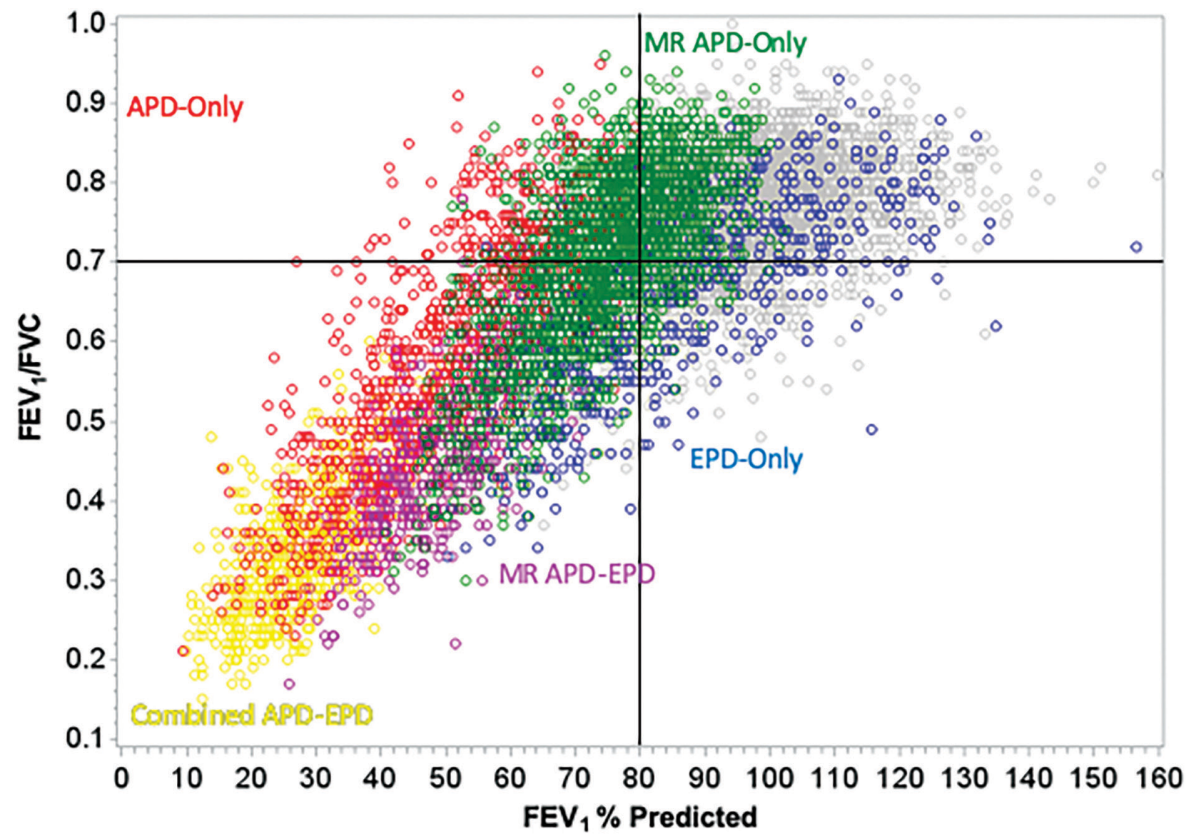

\begin{abstract}
High-risk airway-predominant (APD-only) is represented in red; high-risk emphysema-predominant (EPD-only) in blue; moderate-risk airway-predominant (MR APD-only) in green. Combined high-risk airway and high-risk emphysema-predominant (combined APD-EPD) is represented in yellow.

Combined moderate-risk airway- with high-risk emphysema-predominant (combined MR APD-EPD) is in magenta. Participants not identified with a high-risk or moderate-risk group are shown in grey. (The separate distributions of each group are in Supplementary Figures S1-S6.) Airway-predominant and emphysema-predominant subtypes represent a range of pulmonary function. Current GOLD criteria would define individuals with an $\mathrm{FEV}_{1} \%$ predicted $>80$ and a $\mathrm{FEV}_{1} / \mathrm{FVC}>0.7$ as normal.
\end{abstract}

$\mathrm{FEV}_{1}=$ forced expiratory volume in 1 second; $\mathrm{FVC}=$ forced vital capacity; GOLD=Global initiative for chronic Obstructive Lung Disease

\section{Intra-Individual Measurement Variability of Pulmonary Function}

Twenty-nine participants in COPDGene ${ }^{\circledR}$ had duplicate visits within 6 months during which full clinical assessment was completed including both replicate CT scans and replicate pulmonary function studies. These participants were used to assess intra-individual measurement variability in repeat spirometry. The coefficient of variation of these pairs (mean $/ 1$ SD) for $\mathrm{FEV}_{1} \%$ predicted $=15.7 \%$ and for $\mathrm{FEV}_{1} / \mathrm{FVC}=10.5 \%$. To reduce the impact of measurement variability on our assessment of progression across GOLD boundaries, we removed individuals whose Phase 1 and Phase $2 \mathrm{FEV}_{1} \%$ predicted was within $15.7 \%$ of $80 \%$ (67.44-92.56\%) or whose Phase 1 and Phase $2 \mathrm{FEV}_{1} / \mathrm{FVC}$ ratio was within $10.5 \%$ of 0.70 (0.6265-0.7735). This resulted in excluding 1755 individuals from the Phase 2 progression analysis. Thus, 2860 individuals with complete data and for whom a change across a GOLD boundary would likely represent disease progression and not just acute changes in pulmonary function were used for the Phase 2 progression analysis. The exclusion of all participants close to the GOLD boundaries from progression analysis will likely result in a conservative estimate of progression that would be expected to underestimate total progression. In addition, disease progression that does not result in crossing a GOLD boundary is not considered in this analysis. Changes in GOLD stage irrespective of intraindividual measurement variability are presented in Figure S7 in the online supplement.

\section{Results}

There were marked differences in all-cause mortality among the different high-risk groups defined by unbiased factor analysis. Five-year all-cause 
mortality for the high-risk and moderate-risk airwaypredominant subtypes (APD-only and MR-APD-only) were $21.6 \%$ and $11.0 \%$ while 5 -year mortality for the high-risk emphysema subtype (EPD-only) was 9\% (Table 1). Participants in the combined APD-EPD group had a 5-year mortality of $47.5 \%$ and those in the combined MR-APD-EPD group had a $23.0 \%$ mortality (Table 1). Participants in the combined high-risk groups were primarily in GOLD 2-4 stages at the time of their Phase 1 visits (Figures 2, and S4 and S6 in the online supplement). The airway-predominant groups have higher mean values of measures of airway disease (WA\%), while the emphysema-predominant groups have higher mean values of measures of emphysema (Table 2).

Of the 1375 participants available for progression analysis in GOLD 0 at Phase 1, 4.2\% converted to PRISm, 6.8\% converted to GOLD 1, and $2.0 \%$ converted to GOLD 2-4 at Phase 2 (5-year follow-up) (Figure 3 ). Individuals in the MR-APD-EPD group converted to PRISm (33.3\%), but not to GOLD 1 (0.0\%). Of individuals in the EPD-only group, $11.8 \%$ converted to GOLD 1, but few converted to PRISm (2.0\%). Conversion to GOLD 2-4 occurred in both the MR-APD-only group (8.8\%) and the APD-only group (2.9\%). The MR-APD-only group was associated with conversion from GOLD 0 to PRISm (OR 11.3, 95\% CI 5.7-22.1) and to GOLD 2-4 (OR 6.0, 95\% CI 2.018.0) after adjustment for age, race, sex and change of smoking status (Table 3). The EPD-only group was associated with conversion from GOLD 0 to GOLD 1 (OR 6.0, 95\% 2.0-18.0) after adjustment (Table 3).

Thirty-six percent of the 179 participants available for progression analysis in PRISm at Phase 1 converted to GOLD 2-4 at Phase 2 (Figure 4). No conversion from PRISm to GOLD 0 or GOLD 1 occurred at Phase 2. A large proportion of individuals in the APD-only group (30.7\%) and the MR-APD-only group (37.8\%) in PRISm at Phase 1 converted to GOLD 2-4 at Phase 2 (Figure 4). While a large proportion of the EPD-only group and the No HR MR group converted to GOLD $2-4$, this is due to the small numbers of individuals in these groups ( $n=3$ and $n=9$, respectively) in PRISm. Due to the small number of individuals in the comparison group (No HR MR) in PRISm, multivariable regression models were not run.

In the 174 participants available for progression analysis in GOLD 1 at Phase 1, 18.4\% converted to GOLD 2-4, $1.2 \%$ converted to PRISm, and $27.6 \%$ converted back to GOLD 0 at Phase 2 (Figure 5 ). Individuals in the EPD-only group converted to GOLD 2-4 (25.0\%) and GOLD 0 (19.4\%). While a large proportion of individuals in the MR-APD-only group converted to GOLD 2-4 (5/8) and GOLD 0 (3/8), this represents small numbers of individuals with this group in GOLD 1 (8 total). The EPD-only group was associated with conversion from GOLD 1 to GOLD 2-4 (OR 2.6, 95\% CI 1.0-6.9), after adjustment (Table 3). All participants progressing from GOLD 1 to GOLD 2-4 were categorized as GOLD 2 at Phase 2, and none progressed to GOLD 3 or 4 in this 5-year interval.

Participants in GOLD 0 and PRISm who have the airway-predominant subtype (combined APD-only and MR-APD-only) at Phase 1 have minimal emphysema (Table 4) but have an elevated WA\% suggesting airway inflammation. A large number of the MR-APD-only group are located in GOLD 0 at Phase 1. Progression of these participants into PRISm at Phase 2 is not associated with development of emphysema on CT scan. When participants with airway-predominant disease initially progress from PRISm to GOLD 2-4, there is only a modest increase in emphysema. These participants appear to develop emphysema only late in the course of their disease as they progress to GOLD 3 and 4 (Table 4). These individuals, who do have a larger percentage of emphysema when in GOLD Stages 3 and 4 , are also associated with a marked increase in 5 -year mortality (Table 1).

In contrast, the participants in the EPD-only group develop CT-quantifiable emphysema early, while in the GOLD 0 stage, and move through GOLD 1 to GOLD 2 with a progressive increase in emphysema (Table 4). High-risk emphysema-predominant participants who also qualify for moderate- or high-risk airwaypredominant disease demonstrate the highest degree of emphysema (Table 4) and the highest mortality (Table 1).

Analysis of 5-year progression of participants in GOLD 2 at Phase 1 was restricted to 252 participants who were considered appropriate for progression analysis after removing participants for whom change in GOLD stage could be attributed to intra-individual measurement variability. Of these 252 participants, 197 were in the MR-APD-only or APD-only groups and 71 (36\%) of these progressed to GOLD 3-4 over 5 years. Forty-eight of the 252 participants were in the EPD-only group and 16 (33\%) of these progressed to GOLD 3-4. After accounting for intra-individual 


\section{Figure 3. Progression in 5 Years to Preserved Ratio-Inpaired Spirometry, Global Initiative for Chronic Obstructive Lung Disease Stage 1 and Stages 2-4 from Global Initiative for Chronic Obstructive Lung Disease Stage 0}

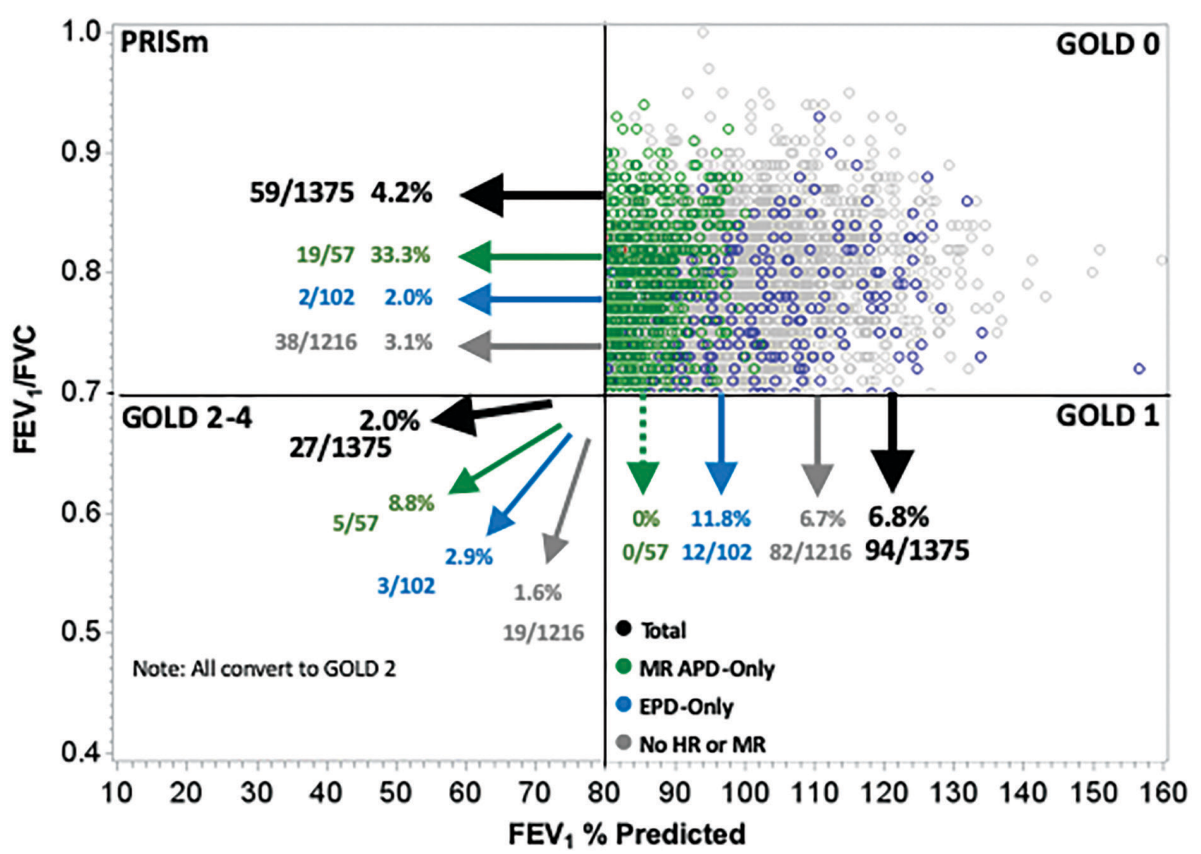

Total progression is represented in black; moderate-risk airway-predominant (MR APD-only) group is represented in green; high-risk emphysema-predominant (EPD-only) group is represented in blue; no high- or moderate-risk group is represented in grey.

Individuals in the MR APD-only group progressed primarily to PRISm or to GOLD 2-4. Individuals in the EPD-only group progressed primarily to GOLD 1. The progression analysis illustrated is for 1375 participants in GOLD 0 at Phase 1 . The figure shows the position of 3562 participants in GOLD 0 in Phase 1. Participants sufficiently close to the GOLD boundaries (2187) that measurement variability could account for change across the boundary were excluded from the progression analysis.

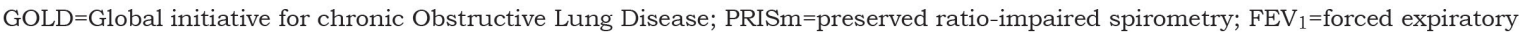
volume in 1 second; FVC=forced vital capacity

\section{Table 3. Odds of Global Initiative for Chronic Obstructive Lung Disease Stage Progression Over 5 Years After Adjustment for Age, Sex, Race and Change in Smoking Status}

\begin{tabular}{l|c|c|c|c} 
& $\begin{array}{c}\text { GOLD 0 to GOLD 1 } \\
\text { OR }(95 \% \mathrm{Cl})\end{array}$ & $\begin{array}{c}\text { GOLD 0 to PRISm } \\
\text { OR }(95 \% \mathrm{Cl})\end{array}$ & $\begin{array}{c}\text { GOLD 0 to GOLD 2-4 } \\
\text { OR }(95 \% \mathrm{Cl})\end{array}$ & $\begin{array}{c}\text { GOLD 1 to GOLD 2-4 } \\
\text { OR }(95 \% \mathrm{Cl})\end{array}$ \\
\hline $\begin{array}{l}\text { No HR or MR group } \\
\text { (reference) }\end{array}$ & 1 & 1 & 1 & 1 \\
\hline APD-Only & NA & NA & NA & NA \\
\hline MR APD-Only & NA & $11.3(5.7-22.1)$ & $6.0(2.0-18.0)$ & $9.2(1.7-49.3)$ \\
\hline EPD-Only & $2.4(1.2-4.6)$ & $0.6(0.1-2.4)$ & $1.9(0.5-6.6)$ & $2.6(1.0-6.9)$ \\
\hline
\end{tabular}

GOLD=Global initiative for chronic Obstructive Lung Disease; PRISm=preserved ratio-impaired spirometry; OR=odds ratio; $\mathrm{CI}=$ confidence interval; $\mathrm{HR}=$ high-risk; $\mathrm{MR}=$ moderate-risk; $\mathrm{APD}$-only=high-risk airway-predominant disease only; NA=not applicable due to small sample size; MR APD-only=moderate-risk airway-predominant disease only; EPD-only=high risk emphysema

measurement error, other changes involving participants in GOLD 2 at Phase 1 included: 6 (2.4\%) went to GOLD 0, 6 (2.4\%) went to GOLD 1, and 7
(2.8\%) went to PRISm at Phase 2.

In a post-hoc analysis, recognizing that we took a conservative approach to deal with intra-individual 


\section{Figure 4. Progression in 5 Years to Global Initiative for Chronic Obstructive Lung Disease Stages 0, 1 and 2-4 from Preserved Ratio-Impaired Spirometry}

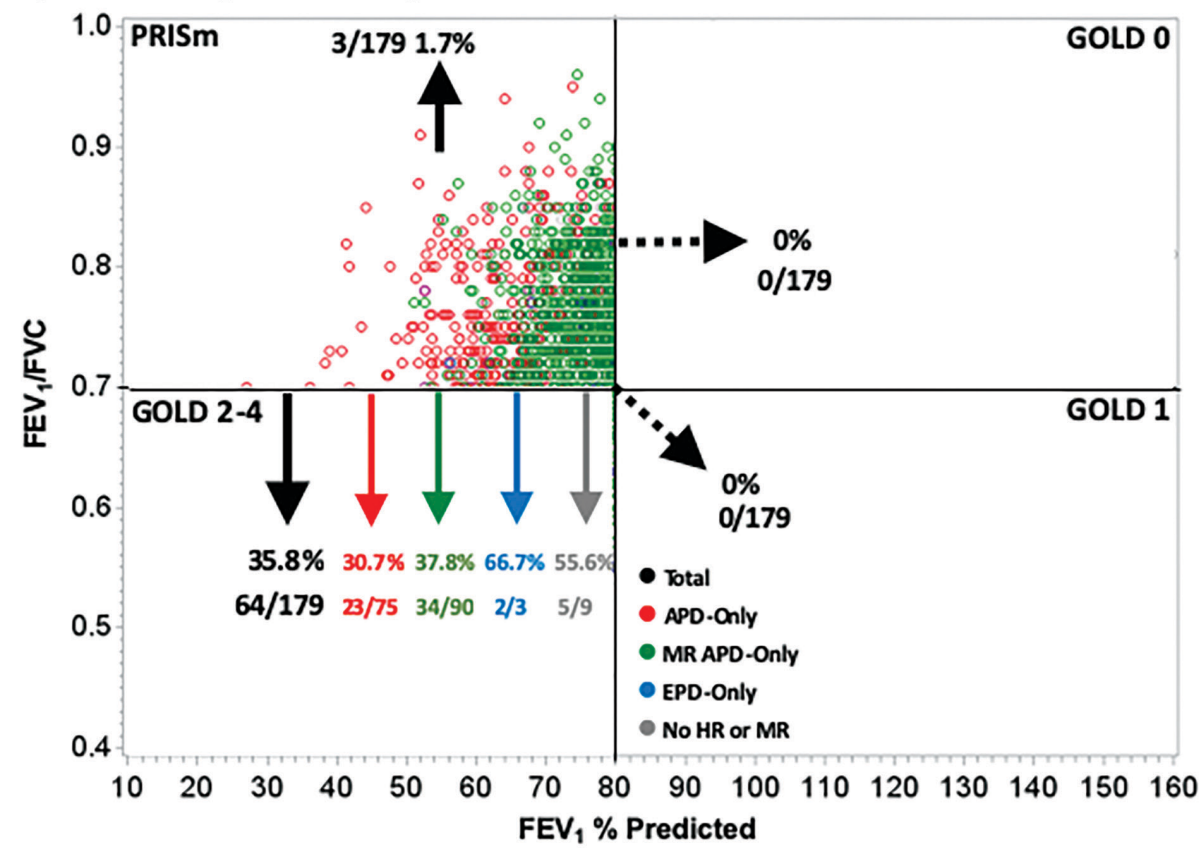

Total progression is represented in black; moderate-risk airway-predominant (MR APD-only) group is represented in green; high-risk airway-predominant (APD-only) group is represented in red; high-risk emphysema-predominant (EPD-only) group is represented in blue; no high- or moderate-risk group is represented in grey.

No individuals converted from PRISm to GOLD 0 or GOLD 1. A total of 37\% of individuals progressed from PRISm to GOLD 2-4. A small number of these individuals progressed directly from PRISm to GOLD 3 or GOLD 4. The progression analysis illustrated is for 179 participants in PRISm at Phase 1. The figure shows the position of 1002 participants in PRISm in Phase 1 . Participants sufficiently close to the GOLD boundaries (823) that measurement variability could account for change across the boundary were excluded from the progression analysis.

PRISm=preserved ratio-impaired spirometry; GOLD $=$ Global initiative for chronic Obstructive Lung Disease; FEV $1=$ forced $_{\text {expiratory }}$ volume in 1 second; FVC=forced vital capacity

variability, we examined total movement across all boundaries (Figure S7 in the online supplement). This analysis had no correction for measurement variability for $\mathrm{FEV}_{1} \%$ predicted and $\mathrm{FEV} \mathrm{V}_{1} / \mathrm{FVC}$ and thus included individuals who changed GOLD stage at Phase 2 due to measurement variability rather than being due to a significant change in disease state. As shown in Figure S7 (online supplement), this analysis identified substantial movement in both directions across GOLD boundaries. However, overall net change in GOLD stages mimicked the progression to worse pulmonary function observed after accounting for intra-individual variability - as shown in Figures 3, 4 and 5.

We assessed the impact of smoking cessation on progression of GOLD stage between Phase 1 and Phase 2 of COPDGene ${ }^{\circledR}$ (Table 5). After accounting for intra-individual measurement variability, $14.8 \%$ of individuals who were current smokers at both Phase 1 and Phase 2 progressed, whereas $6.8 \%$ of individuals who were former smokers at both Phase 1 and Phase 2 progressed in GOLD spirometry stage between Phase 1 and Phase $2(p<0.0001)$. As shown in Table 5 , the primary gain for smoking cessation in terms of progression across GOLD stage was for individuals in GOLD 0 or GOLD 1. Of current smokers in GOLD 0, $18.0 \%$ progressed to PRISm, GOLD 1 or GOLD 2-4. Of former smokers in GOLD 0, only $10.2 \%$ progressed to PRISm, GOLD 1 or GOLD 2-4. This contrasts with the findings for participants demonstrating PRISm physiology at baseline. Of current smokers in PRISm, $37 \%$ progressed to GOLD $2-4$, and $35.9 \%$ of former smokers in PRISm progressed to GOLD 2-4.

\section{Discussion}

Previous work has defined 2 underlying pathophysiologic processes related to pulmonary 


\section{Figure 5. Progression in 5 Years to Preserved Ratio-Impaired Spirometry, Global Initiative for Chronic Obstructive Lung Disease Stages 2-4 and 0 from Global Initiative for Chronic Obstructive Lung Disease Stage 1}

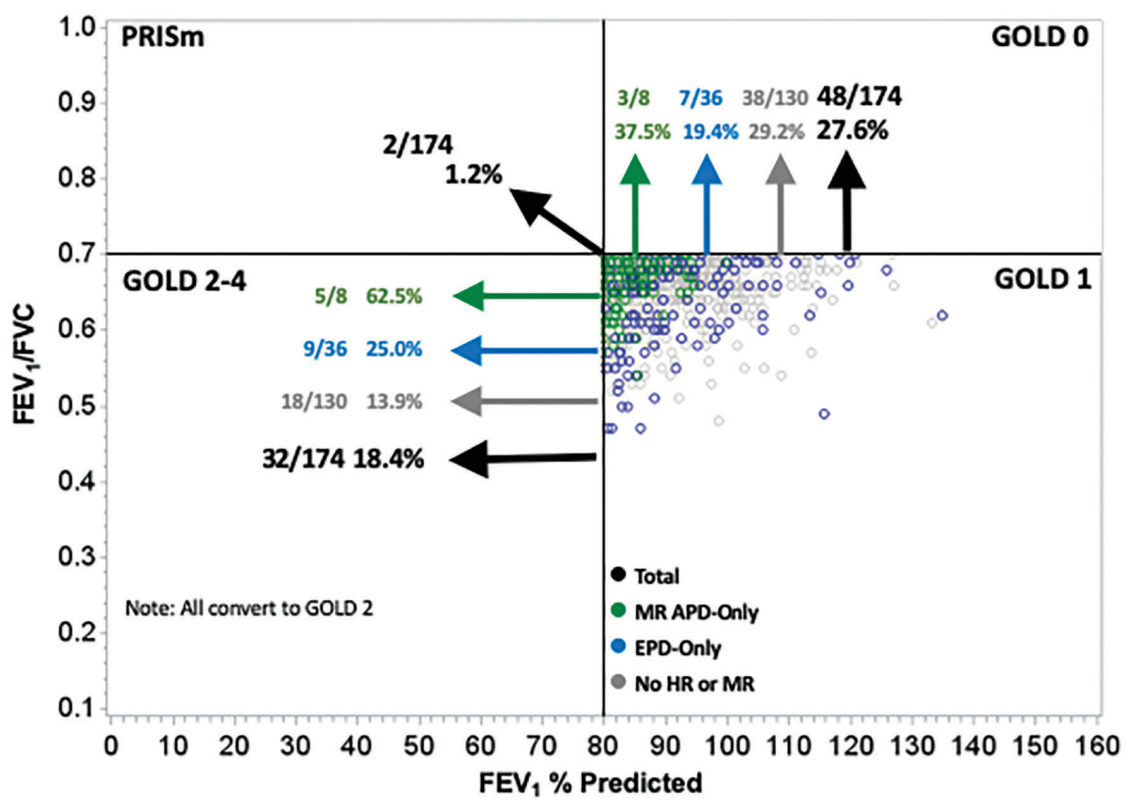

Total progression is represented in black; moderate-risk airway-predominant group (MR APD-only) is represented in green; high-risk emphysema-predominant group (EPD-only) is represented in blue; no high- or moderate-risk group is represented in grey.

All 3 groups represented in GOLD 1 showed significant progression to GOLD 2. None of the GOLD 1 participants progressed to GOLD 3 or 4. The progression analysis illustrated is for 174 participants in GOLD 1 at Phase 1 . The figure shows the position of 647 participants in GOLD 1 in Phase 1. Participants sufficiently close to the GOLD boundaries (473) that measurement variability could account for change across the boundary were excluded from the progression analysis.

GOLD=Global initiative for chronic Obstructive Lung Disease; PRISm=preserved ratio-impaired spirometry; FEV ${ }_{1}=$ forced expiratory volume in 1 second; $\mathrm{FVC}=$ forced vital capacity

function: airway-predominant disease and emphysema-predominant disease which are associated with differential risk of mortality. ${ }^{15}$ We now find that individuals with excess risk of mortality based on these disease axes demonstrate unique patterns of disease progression through GOLD stages towards more severe COPD. We have chosen to use a conservative approach to assess the magnitude of progression by excluding participants so sufficiently close to the GOLD boundaries that intra-individual measurement variability could account for change. We also did not assess progression within a GOLD stage that does not cross a boundary. Our results, by design, likely underestimate the true magnitude of progression. We recognize that participants close to a boundary are at greatest risk for progressing across that boundary. The current analysis is directed at evaluating the relative progression of the 6 subtypes identified by unbiased factor analysis and mortality risk.

Participants with airway-predominant disease or emphysema-predominant disease associated with excess mortality are identified within GOLD 0 and demonstrate higher levels of conversion to more advanced GOLD stages than individuals without 1 of these 2 underlying disease processes. The primary pattern for progression of the airway-predominant disease is from GOLD 0 to PRISm and from PRISm to GOLD 2-4 while the pattern for progression of emphysema-predominant disease is primarily from GOLD 0 to GOLD 1 and from GOLD 1 to GOLD 2-4. Fifty percent of the entire cohort (8157 individuals with a substantial smoking history) are associated with moderate or high-risk airway-predominant disease (combining APD-only, MR-APD-onlyand the combined APD-EPD groups [Figures 1 and 2]). Participants with airway-predominant disease appear to be on a pathway of disease progression that has not previously been well appreciated and that is associated with a markedly elevated 5-year all-cause mortality. Twenty percent of the entire cohort was identified as having high-risk emphysema-predominant disease (Figures 1 and 2) with a different pattern of disease progression 


\section{Table 4. Characteristics of Subtype by Global Initiative for Chronic Obstructive Lung Disease Grade at Phase 1}

\begin{tabular}{|c|c|c|c|c|c|c|}
\hline Subtype & GOLD 0 & PRISm & GOLD 1 & GOLD 2 & GOLD 3 & GOLD 4 \\
\hline \multicolumn{7}{|c|}{ Airway Predominant (High + Moderate Risk) } \\
\hline $\mathrm{n}=4079$ (Phase 1) & 572 & 879 & 83 & 1167 & 917 & 461 \\
\hline \% Emphysema (\%LAA < -950 HU) & $0.3 \pm 0.5$ & $1.0 \pm 1.6$ & $0.7 \pm 0.7$ & $5.6 \pm 6.8$ & $16.6 \pm 12.4$ & $27.2 \pm 14.1$ \\
\hline WA $\%$ & $52.7 \pm 7.4$ & $54.7 \pm 7.7$ & $53.7 \pm 7.6$ & $55.7 \pm 7.5$ & $56.7 \pm 7.6$ & $56.1 \pm 7.4$ \\
\hline \multicolumn{7}{|l|}{ Emphysema Predominant } \\
\hline $\mathrm{n}=1631$ (Phase 1) & 213 & 23 & 113 & 410 & 502 & 370 \\
\hline \% Emphysema (\%LAA < -950 HU) & $7.9 \pm 4.1$ & $9.1 \pm 6.9$ & $13.5 \pm 7.2$ & $17.8 \pm 8.4$ & $24.9 \pm 10.2$ & $31.8 \pm 11.6$ \\
\hline WA\% & $42.0 \pm 5.9$ & $48.5 \pm 6.4$ & $45.0 \pm 5.9$ & $50.8 \pm 6.9$ & $53.7 \pm 6.7$ & $54.8 \pm 7.0$ \\
\hline \multicolumn{7}{|l|}{ No Subtype } \\
\hline $\mathrm{n}=3524$ (Phase 1) & 2777 & 100 & 451 & 196 & 0 & 0 \\
\hline \% Emphysema (\%LAA < -950 HU) & $1.8 \pm 2.0$ & $3.7 \pm 2.6$ & $3.9 \pm 3.2$ & $6.2 \pm 3.8$ & NA & NA \\
\hline WA $\%$ & $46.5 \pm 6.9$ & $48.0 \pm 5.8$ & $48.7 \pm 6.8$ & $49.9 \pm 6.8$ & NA & NA \\
\hline
\end{tabular}

There is overlap in the airway-predominant and emphysema-predominant numbers due to the individuals in the combined airway-predominant and emphysema-predominant groups (high-risk airway- and emphysema-predominant and moderate-risk airway-predominant and high-risk emphysema predominant groups).

GOLD=Global initiative for chronic Obstructive Lung Disease; PRISm=preserved ratio-impaired spirometry; \%LAA=percentage of low attenuation area; $\mathrm{HU}=$ Hounsfield units; $\mathrm{WA} \%=$ wall area percentage for segmental airways.

and who also show a significant elevation in 5-year all-cause mortality. Identification of these 2 distinct patterns of COPD progression offer opportunities to identify participants for unique interventions to disrupt specific underlying disease processes related to pulmonary function decline.

Individuals in the MR-APD-only group represent 4.1\% of GOLD 0. One-third (33.3\%) of these progressed to PRISm within 5 years, while none progressed to GOLD 1, and a small proportion progressed to GOLD 2-4 (5/57). The vast majority of individuals in PRISm have airway-predominant disease (167/179) with only $1.7 \%$ having emphysema-predominant disease and $2.8 \%$ being in the no high-risk group category. Of the COPDGene ${ }^{\circledR}$ participants who were in PRISm at baseline, greater than $1 / 3$ progressed to GOLD 2-4 over 5 years while none moved into GOLD 0 or GOLD 1. Thus, PRISm represents an advanced, progressive disease state associated with airway-predominant disease that shows no improvement in pulmonary function in 5 years. (Note: PRISm participants in the COPDGene ${ }^{\circledR}$ cohort are heavy current and former smokers, and participants with a clinical diagnosis of interstitial lung disease were excluded from this cohort.)

Participants with airway-predominant disease who progressed from GOLD 0 to PRISm and from PRISm to
GOLD 2-4 over 5 years had minimal to no detectable emphysema and no significant change in emphysema from Phase 1 to Phase 2 (Table 5). Participants with airway-predominant disease who were in GOLD 2-4 at Phase 1 had substantial amounts of emphysema with marked differences between GOLD stages 2, 3 and 4 (Table 4). This suggests that development and progression of emphysema in the airway-predominant groups is late in the disease process. In comparison, the high-risk emphysema-predominant group had significant emphysema early in their disease process (i.e., in GOLD 0) at Phase 1. The high-risk emphysemapredominant group was associated with progressively greater amounts of emphysema associated with being in GOLD stages 1, 2, 3 and 4 in the Phase 1 crosssectional analysis (Table 4).

Individuals at high risk of mortality associated with emphysema-predominant disease represent $7.4 \%$ of GOLD 0 participants. Participants with emphysemapredominant disease appear to be relatively stable within GOLD 0 with only $16.7 \%$ changing GOLD stage over 5 years ( $11.8 \%$ converted to GOLD 1, 2.0\% converted to PRISm, and 2.9\% converted to GOLD 2-4). GOLD 1 appears to be a relatively unstable stage with a total of $46.0 \%$ changing GOLD stage over 5 years (18.4\% progressed to GOLD 2-4 and $27.6 \%$ moved back to GOLD 0). The majority of GOLD 1 participants 


\section{Table 5. Progression Between Phase 1 and Phase 2 by Smoking Status}

\begin{tabular}{l|r|rr}
\multicolumn{4}{c}{ Smoking Status at Both Phase 1 and Phase 2 of COPDGene ${ }^{\circledR}$} \\
\hline \\
GOLD O to PRISm & Current Smoker $(\mathrm{n}=1226)$ & Former Smoker $(\mathrm{n}=1734)$ & $p$-value \\
\hline GOLD O to GOLD 1 & $36 / 655(5.5 \%)$ & $18 / 757(2.4 \%)$ & 0.002 \\
\hline GOLD O to GOLD 2-4 & $61 / 655(9.3 \%)$ & $49 / 757(6.5 \%)$ & 0.047 \\
\hline GOLD 1 to GOLD 2-4 & $21 / 655(3.2 \%)$ & $10 / 757(1.3 \%)$ & 0.02 \\
\hline PRISm to GOLD 2-4 & $18 / 77(23.4 \%)$ & $13 / 95(13.7 \%)$ & 0.10 \\
\hline
\end{tabular}

$p$-value represents differences between smoking groups, calculated with the chi-square test.

Participants who changed smoking status (current to former or former to current) between Phase 1 and Phase 2 are not included in the above table.

GOLD=Global initiative for chronic Obstructive Lung Disease; PRISm=preserved ratio-impaired spirometry

changing GOLD stage were categorized as not being in a high-risk group. Participants with emphysemapredominant disease showed significant progression from GOLD 1 to GOLD 2-4 (25.0\%, OR=2.6); however, $19.4 \%$ of GOLD 1 participants with emphysemapredominant disease moved back to GOLD 0.

Smoking cessation was found to have a substantial impact on decreasing the probability of an individual progressing in GOLD stage when smoking cessation occurred prior to progression to PRISm or to GOLD 2-4 (Table 5). All groups of participants in the COPDGene ${ }^{\circledR}$ cohort had a history of heavy smoking. Former smokers in GOLD 0 or GOLD 1 at baseline had a marked decrease in risk of progression over the next 5 years. In contrast, former smokers who were in PRISm at baseline carried the same risk of progression to GOLD 2-4 as did current smokers. This suggests that heavy smoking, when associated with airwaypredominant disease and associated with progression into PRISm physiology, carries a poor prognosis even after smoking cessation.

The purpose of the current study was to investigate the progression of underlying pathophysiologic disease processes through clinically-defined categories of COPD (GOLD spirometry stages) over 5 years. Key strengths of the study are a large, well characterized cohort of current and former smokers with both CT scans and pulmonary physiology measures. In addition, intra-individual measurement variability of pulmonary function was derived from within the COPDGene ${ }^{\circledast}$ study. This allowed for removing noise associated with the well-recognized variability of the pulmonary function test. ${ }^{24,25}$ Our post-hoc analysis that eliminated consideration of intra-individual measurement variability showed a substantial amount of apparent movement of individuals across GOLD boundaries. However, net change in GOLD classification in this post-hoc analysis (Figure S7, online supplement) was found to closely mimic the primary findings of progression of pulmonary function to higher (worse) GOLD stages (Figures 3-5). Although by design, conservative estimates of progression were obtained, and it should be recognized that this approach will mainly identify rapid progressors. Further analyses examining movement within GOLD spirometry grade and including data from the ongoing COPDGene ${ }^{\circledR}$ Phase 3 will enable a better understanding of progression within the airwaypredominant and emphysema-predominant disease subtypes.

Much previous work in COPD has shown that individuals with a low $\mathrm{FEV}_{1} / \mathrm{FVC}$ are at risk of rapid progression and loss of $\mathrm{FEV}_{1}$, and that these patients also have a poor overall prognosis. ${ }^{26}$ The question of disease progression in less advanced phases of lung disease remains open. The Lung Health Study looked at differences between those who quit smoking compared to those who continued to smoke in a cohort of smokers with largely mild to moderate disease, finding that continued smokers declined steadily in mean $\mathrm{FEV}_{1}$ at a rate of approximately $60 \mathrm{ml} /$ year while quitters declined at approximately half that rate. ${ }^{27}$ There was no consideration in either of these studies of high-risk disease subtypes nor patterns of progression from smokers without defined spirometric disease.

This study identified 2 discrete patterns of disease progression from GOLD 0 to GOLD 2-4 among smokers. One pathway is characterized by those 
with airway-predominant disease and demonstrates an initial decline in both $\mathrm{FEV}_{1} \%$ predicted and FVC which would move them over 5 years toward or into PRISm physiology. A high percentage of COPDGene ${ }^{\circledR}$ participants who were in PRISm at baseline were found to move into GOLD 2-4 after 5 years of observation. This was associated with a further decline in $\mathrm{FEV}_{1} \%$ predicted and a decline in FEV $1 / F V C$. A second pathway is defined by an initial 5-year decline in the $\mathrm{FEV}_{1} / \mathrm{FVC}$ ratio from GOLD 0 to GOLD 1 and is the major pathway seen among those with emphysemapredominant disease. While GOLD 1 appears to be a relatively unstable stage, the emphysemapredominant subtype shows an overall subsequent progression to GOLD 2-4. The characterization of these discrete patterns of disease progression associated with underlying pathophysiologic disease processes offers a unique opportunity for personalized targeted intervention to prevent disease progression and pulmonary function decline among smokers.

\section{Acknowledgements}

\section{Author Contributions}

All authors contributed to study design, critical review of the manuscript for important intellectual content, and interpretation of data. Statistical analysis was done by KAY, GLK, JEH, MJS, and EAR.

\section{Declaration of Interest}

EKS has received grant and travel support from GlaxoSmithKline. All other authors declare they have no conflicting interests. 


\section{References}

1. Friedlander AL, Lynch D, Dyar LA, Bowler RP. Phenotypes of chronic obstructive pulmonary disease. COPD. 2007; 4(4):355384. doi: https://doi.org/10.1080/15412550701629663

2. Rabe KF, Hurd S, Anzueto A, et al. Global strategy for the diagnosis, management, and prevention of chronic obstructive pulmonary disease: GOLD executive summary. Am J Respir Crit Care Med. 2007;176(6):532-555.

doi: https://doi.org/10.1164/rccm.200703-456SO

3. Menezes AM, Wehrmeister FC, Perez-Padilla R, et al. The PLATINO study: description of the distribution, stability, and mortality according to the Global Initiative for Chronic Obstructive Lung Disease classification from 2007 to 2017. Int J Chron Obstruct Pulmon Dis. 2017;12:1491-1501. doi: https://doi.org/10.2147/COPD.S136023

4. Agusti A, Edwards LD, Celli B, et al. Characteristics, stability and outcomes of the 2011 GOLD COPD groups in the ECLIPSE cohort. Eur Respir J. 2013; 42(3):636-646.

doi: https://doi.org/10.1183/09031936.00195212

5. Goossens LM, Leimer I, Metzdorf N, et al. Does the 2013 GOLD classification improve the ability to predict lung function decline, exacerbations and mortality: a post-hoc analysis of the 4-year UPLIFT trial. BMC Pulm Med. 2014; 14:163.

doi: https://doi.org/10.1186/1471-2466-14-163

6. Kim J, Yoon HI, Oh YM, et al. Lung function decline rates according to GOLD group in patients with chronic obstructive pulmonary disease. Int J Chron Obstruct Pulmon Dis. 2015;18191827. doi: https://doi.org/10.2147/COPD.S87766

7. Regan EA, Hokanson JE, Murphy JR, et al. Genetic epidemiology of COPD (COPDGene) study design. COPD. 2010;7(1):32-43. doi: https://doi.org/10.3109/15412550903499522

8. Wan ES, Castaldi PJ, Cho MH, et al. Epidemiology, genetics, and subtyping of preserved ratio impaired spirometry (PRISm) in COPDGene. Respir Res. 2014; 5:89.

doi: https://doi.org/10.1186/s12931-014-0089-y

9. Vestbo J, Lange P. Can GOLD Stage 0 provide information of prognostic value in chronic obstructive pulmonary disease? Am J Respir Crit Care Med. 2002;166(3):329-332. doi: https://doi.org/10.1164/rccm.2112048

10. Lindberg A, Bjerg A, Ronmark E, et al. Prevalence and underdiagnosis of COPD by disease severity and the attributable fraction of smoking. Report from the Obstructive Lung Disease in Northern Sweden Studies. Respir Med. 2006;100(2):264-272. doi: https://doi.org/10.1016/j.rmed.2005.04.029

11. Lundback B, Lindberg A, Lindstrom M, et al. Not 15 but 50\% of smokers develop COPD? Report from the Obstructive Lung Disease in Northern Sweden Studies. Respir Med. 2003; 97(2):115-22. doi: https://doi.org/10.1053/rmed.2003.1446
12. Wan ES, Fortis S, Regan EA, et al. Longitudinal phenotypes and mortality in preserved ratio impaired spirometry in the COPDGene Study. Am J Respir Crit Care Med. 2018;198(11): 1397-1405. doi: https://doi.org/10.1164/rccm.201804-0663OC

13. Arjomandi M, Zeng S, Barjaktarevic I, et al. Radiographic lung volumes predict progression to COPD in smokers with Preserved Spirometry in SPIROMICS. Eur Respir J. 2019;In press. doi: https://doi.org/10.1183/13993003.02214-2018

14. Zeng S, Tham A, Bos B, et al. Lung volume indices predict morbidity in smokers with preserved spirometry. Thorax. 2019; 74(2):114-124.

doi: https://doi.org/10.1136/thoraxjnl-2018-211881

15. Kinney GL, Santorico SA, Young KA, et al. Identification of chronic obstructive pulmonary disease axes that predict allcause mortality: The COPDGene Study. Am J Epidemiol. 2018; 187(10):2109-2116. doi: https://doi.org/10.1093/aje/kwy087

16. Young KA, Regan EA, Han MK, et al and the COPDGene Investigators. Subtypes of COPD have unique distributions and differential risk of mortality. Chronic Obstr Pulm Dis. 2019;6(5): 400-413. doi: https://doi.org/10.15326/jcopdf.6.5.2019.0150

17. Regan EA, Lynch DA, Curran-Everett D, et al. Clinical and radiologic disease in smokers with normal spirometry. JAMA Intern Med. 2015; 175(9):1539-1549.

doi: https://doi.org/10.1001/jamainternmed.2015.2735

18. Standardization of Spirometry, 1994 Update. American Thoracic Society. Am J Respir Crit Care Med. 1995; 152(3):1107-1136. doi: https://doi.org/10.1164/ajrccm.152.3.7663792

19. ATS statement: guidelines for the six-minute walk test. Am J Respir Crit Care Med. 2002;166(1):111-117. doi: https://doi.org/10.1164/ajrccm.166.1.at1102

20. Stewart JI, Moyle S, Criner GJ, et al. Automated telecommunication to obtain longitudinal follow-up in a multicenter cross-sectional COPD study. COPD. 2012; 9(5):466472. doi: https://doi.org/10.3109/15412555.2012.690010

21. Charbonnier JP, Pompe E, Moore C, et al. Airway wall thickening on CT: relation to smoking status and severity of COPD. Respir Med. 2019;146:36-41.

doi: https://doi.org/10.1016/j.rmed.2018.11.014

22. Patel BD, Coxson HO, Pillai SG, et al. Airway wall thickening and emphysema show independent familial aggregation in chronic obstructive pulmonary disease. Am J Respir Crit Care Med. 2008;178(5):500-505.

doi: https://doi.org/10.1164/rccm.200801-0590C

23. Bhatt SP, Washko GR, Hoffman EA, et al. Imaging advances in chronic obstructive pulmonary disease. insights from the Genetic Epidemiology of Chronic Obstructive Pulmonary Disease (COPDGene) Study. Am J Respir Crit Care Med. 2019;199(3):286-301.

doi: https://doi.org/10.1164/rccm.201807-1351SO 
24. Aaron SD, Tan WC, Bourbeau J, et al. Diagnostic instability and reversals of chronic obstructive pulmonary disease diagnosis in individuals with mild to moderate airflow obstruction. Am J Respir Crit Care Med. 2017;196(3):306-314.

doi: https://doi.org/10.1164/rccm.201612-2531OC

25. Vestbo J, Edwards LD, Scanlon PD, et al. Changes in forced expiratory volume in 1 second over time in COPD. New Engl J Med. 2011;365(13):1184-1192.

doi: https://doi.org/10.1056/NEJMoa1105482

26. Burrows B, Knudson RJ, Camilli AE, et al. "The horse-racing effect" and predicting decline in forced expiratory volume in one second from screening spirometry. Am Rev Respir Dis. 1987; 135(4):788-93. doi: https://doi.org/10.1164/arrd.1987.135.4.788

27. Anthonisen NR, Connett JE, Murray RP. Smoking and lung function of Lung Health Study participants after 11 years. Am J Respir Crit Care Med. 2002;166(5):675-679.

doi: https://doi.org/10.1164/rccm.2112096 